\title{
Influence of the Light Schedule on the Toxicity of Amitriptyline in Chick Embryos
}

\author{
Yuji Yoshiyama, ${ }^{* a}$ Takashi SugiYama, ${ }^{b}$ and Motoko Kanke ${ }^{a}$ \\ ${ }^{a}$ Division of Clinical Pharmacy, Kyoritsu University of Pharmacy; 1-5-30 Shibakoen, Minato-ku, Tokyo 105-8512, \\ Japan: and ${ }^{b}$ Division of Pathophysiology, Center for Clinical Pharmacy and Clinical Sciences, Kitasato University School \\ of Pharmaceutical Sciences; Minato-ku, Tokyo 108-8641, Japan. Received August 20, 2003; accepted October 20, 2003
}

\begin{abstract}
The cardiac toxicity of amitriptyline and the effect of the light schedule on it were studied in chick embryos. Fertilized eggs of White Leghorns were incubated under dark conditions and investigated, on two occasions, in the light phase and in the dark phase. Amitriptyline was injected into the air sac of fertilized eggs on the 16th day of incubation. Electrocardiograms were recorded 0 to $60 \mathrm{~min}$ after the injection. After the administration of amitriptyline $1 \mathrm{mg} / \mathrm{egg}$ in the light phase, the heart rate did not differ compared with that in controls. However, the heart rate was significantly decreased by the administration of amitriptyline $2.5 \mathrm{mg} / \mathrm{egg}$ and $5 \mathrm{mg} / \mathrm{egg}$ in the light phase. The heart rate was significantly decreased by the administration of amitriptyline $1 \mathrm{mg} / \mathrm{egg}$ under dark conditions. In addition, arrhythmia was produced by the administration of amitriptyline under dark conditions. These findings indicate that manipulation of the light schedule has a marked influence on the toxicity of amitriptyline in chick embryos.
\end{abstract}

Key words light schedule; cardiotoxicity; amitriptyline; chick embryo; electrocardiogram

Circadian-dependent changes in pharmacologic effects have been demonstrated for a variety of drugs in humans ${ }^{1,2)}$ and rodents. ${ }^{3,4)}$ The light schedule is undoubtedly an important determinant in the circadian rhythm of drug actions. Amitriptyline is known to alter myocardial function as manifested by electrocardiogram (ECG) changes. Although tricyclic antidepressants may have cardiovascular effects at therapeutic doses, they are generally thought to be dose related, and widening of the QRS complex is reported to correlate well with the severity of toxicity following acute overdose. $^{5,6)}$

With the recent concern for animal rights, experimental studies using mammals have been limited in number and methods. Thus, based on social acceptance, experimental studies using chick embryos have drawn attention. Chick embryos have been widely used in pharmacologic and toxicologic experiments for evaluating drug action on the fetus. ${ }^{7-9)}$ To develop alternative methods, we have studied the biologic effects of drugs on the cardiovascular system of chick embryos using physiologic techniques. ${ }^{10-14)}$

The present study evaluated the effect of amitriptyline on the heart and the effect of the light schedule on the toxicity of amitriptyline in chick embryos.

\section{MATERIALS AND METHODS}

Fertilized eggs of White Leghorns (Omiya Poultry Laboratory, Saitama, Japan) were incubated at $37.5 \pm 0.2{ }^{\circ} \mathrm{C}$ at a relative humidity of about $65 \%$, turned automatically every hour, and under dark conditions. An experiment was performed in the light phase (under ta fluorescent lamp, 450 lux) or in the dark phase (under a red safety lamp, 12 lux) for $2 \mathrm{~h}$ after acclimatization.

Amitriptyline hydrochloride (Banyu Pharmaceutical Co., Ltd., Tokyo, Japan) was used for the treatment. Amitriptyline $1 \mathrm{mg} / \mathrm{egg}, 2.5 \mathrm{mg} / \mathrm{egg}$, or $5 \mathrm{mg} / \mathrm{egg}$ was injected into the air sac of each fertilized egg in the light phase. After each drug injection in the light phase, the heart rate was measured. After the administration of amitriptyline $1 \mathrm{mg} / \mathrm{egg}$ in the light phase or dark phase, the heart rate was also measured.

ECGs were recorded 0 to $60 \mathrm{~min}$ after drug injection, and heart rate was determined based on $\mathrm{R}-\mathrm{R}$ intervals. Changes in heart rate were expressed as mean percentage changes in the drug-treated groups compared with the matched control.

Four small holes were made at 90-degree intervals on "the equator," one small hole on "the south pole," and one small hole on "the north pole" of each fertilized egg using an electric drill and sealed with paraffin $\left(\mathrm{mp} 60^{\circ} \mathrm{C}\right)$. Specially designed needle electrodes were inserted into the appropriate holes of the equator and the south pole. Two needles on the equator were used as a bipolar lead from the embryonic heart, and the needle on the south pole was used as a ground lead. These needles were connected to a memory oscilloscope (VC-11, Nihon Koden Co., Tokyo, Japan). ECGs were recorded as bipolar waves between two needles on a recorder (PowerLab System, ADInstruments Japan Co., Tokyo, Japan) (Fig. 1).

The data were analyzed using one-way analysis of variance. If there was a significant difference among the groups, a multiple-comparison test was conducted (Tukey's test). The fiducial limit of 0.05 , two-tailed, was used as the criterion to determine significance.

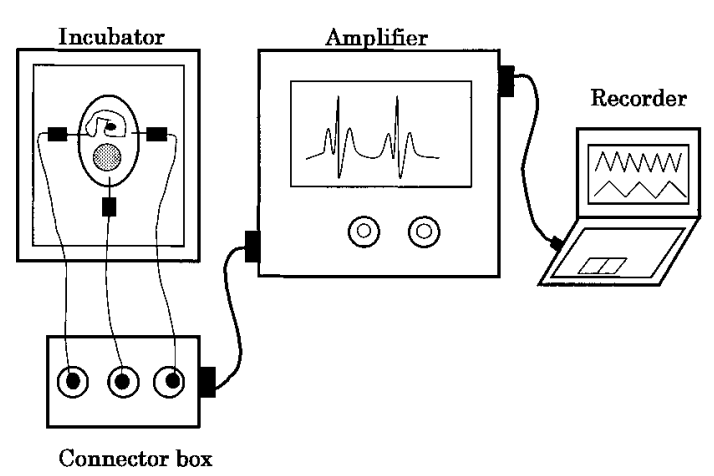

Fig. 1. Schema of ECG Recording System for Chick Embryos in the Egg Shell 


\section{RESULTS}

In the untreated state, the heart rate of the chick embryos

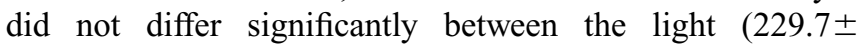
$10.0 \mathrm{bpm})$ and dark $(211.3 \pm 9.0 \mathrm{bpm})$ conditions. Moreover, there were no significant differences in the growth rate of the chick embryos on each light schedule, and the body weights of chick embryos gradually increased with the day of incubation.

After the administration of amitriptyline $1 \mathrm{mg} / \mathrm{egg}$ in the light phase, the heart rate did not differ compared with that of controls. However, the heart rate was significantly decreased with the administration of amitriptyline $2.5 \mathrm{mg} / \mathrm{egg}$ and $5 \mathrm{mg} / \mathrm{egg}$ in the light phase (Fig. 2). The heart rate was also significantly decreased by the administration of amitriptyline $1 \mathrm{mg} /$ egg under the dark conditions (Fig. 3). In addition, arrhythmia was produced by the administration of amitriptyline under dark conditions (Fig. 4).

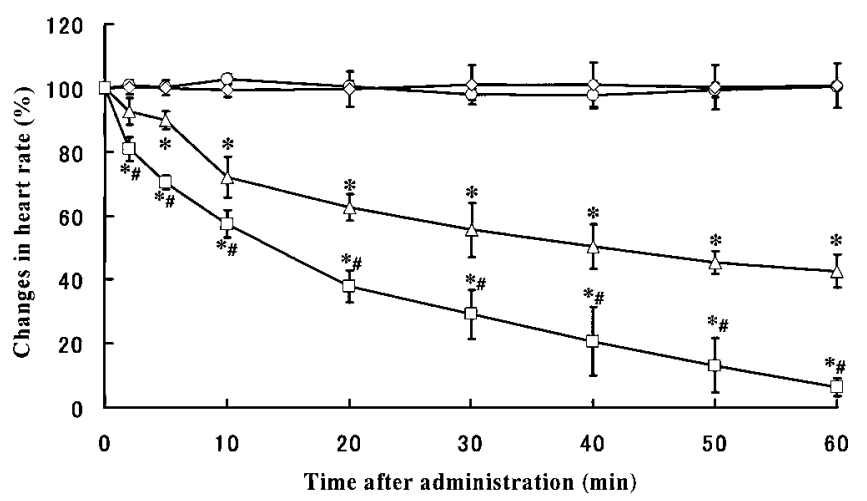

Fig. 2. Changes in Heart Rate of Chick Embryos after Administration of Amitriptyline in the Light Phase

Saline $(\bigcirc)$, or amitriptyline $1 \mathrm{mg} / \mathrm{egg}(\diamond), 2.5 \mathrm{mg} / \mathrm{egg}(\triangle)$, or $5 \mathrm{mg} / \mathrm{egg}(\square)$ was injected into the air sac of fertile eggs in the light phase. Changes in heart rate are expressed as mean percentage changes in the drug-treated groups compared with the time-matched control. Each point represents the mean and S.D. (bar) of 6 eggs. * Significantly different from saline group, $p<0.05$. \# Significantly different from amitriptyline $1 \mathrm{mg} / \mathrm{egg}$ group, $p<0.05$. The heart rates of chick embryos before each drug injection were saline, $238 \pm 8 \mathrm{bpm}$; amitriptyline $1 \mathrm{mg} / \mathrm{egg}, 229 \pm 9 \mathrm{bpm}$; amitriptyline $2.5 \mathrm{mg} / \mathrm{egg}, 236 \pm 15 \mathrm{bpm}$; amitriptyline $5 \mathrm{mg} / \mathrm{egg}, 234 \pm 15 \mathrm{bpm}$.

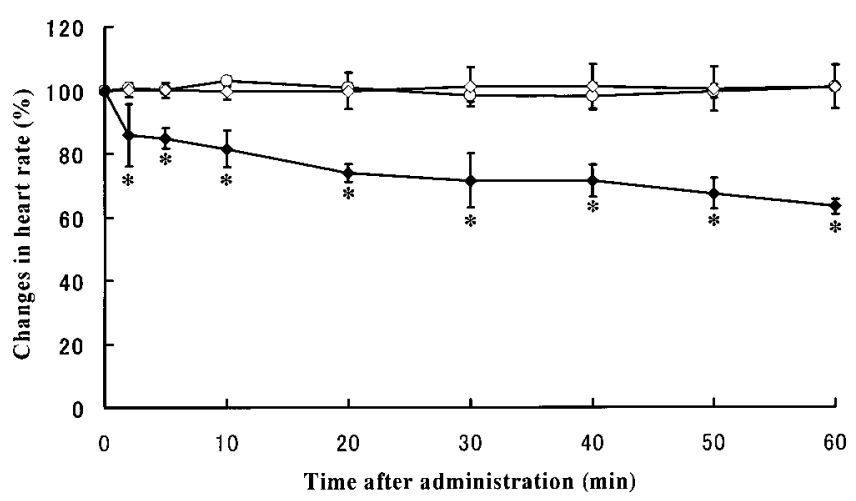

Fig. 3. Changes in Heart Rate of Chick Embryos after Administration of Amitriptyline in the Light or Dark Phase

Saline $(\bigcirc)$, or amitriptyline $1 \mathrm{mg} /$ egg in the light phase $(\diamond)$ or in the dark phase $(\diamond$ was injected into the air sac of fertile eggs on the 16th day of incubation. Changes in heart rate are expressed as mean percentage changes in drug-treated groups compared with the time-matched control. Each point represents the mean and S.D. (bar) of 6 eggs. Significantly different from the amitriptyline $1 \mathrm{mg} / \mathrm{egg}$ under light conditions group, $p<0.05$.
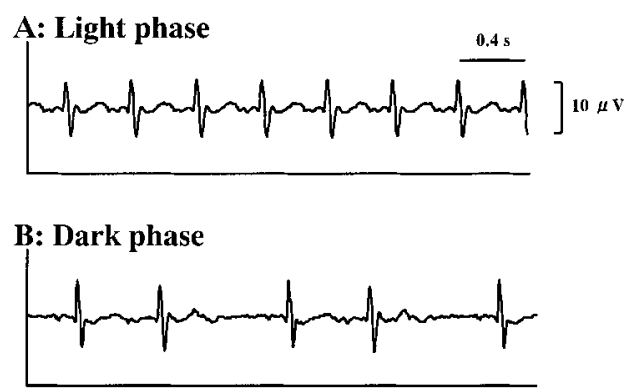

Fig. 4. ECG Tracings in Chick Embryos Treated with Amitriptyline

A: After the injection of amitriptyline $1 \mathrm{mg} / \mathrm{egg}$ in the light phase, B: Arrhythmia occurred after the injection of amitriptyline $1 \mathrm{mg} / \mathrm{egg}$ in the dark phase.

\section{DISCUSSION}

Amitriptyline is a tricyclic antidepressant and may be used in the therapy of depression. The mechanism of action of amitriptyline is thought to be inhibition of the membrane pump mechanism responsible for uptake of norepinephrine and serotonin in adrenergic and serotonergic neurons. ${ }^{15)}$ Newer classes of antidepressants are not more effective than amitriptyline.

It was demonstrated that imipramine has quinidine-like properties, probably by a direct effect on myocardial cells. ${ }^{16}$ ) Caution should be taken when prescribing antidepressants to the elderly as they appear to be less tolerant to their cardiac effects.

The cardiotoxicity of amitriptyline was demonstrated in chick embryos. Amitriptyline led to QTc interval prolongation in the ECGs. After the drug was injected into the air sac of each fertilized egg, it accumulated in the eggshell. Therefore the heart rate may be decreased time dependently. This time-dependent effect of the drug on the heart rate should be investigated further.

The present results indicate that our ECG recording system using chick embryos may be used as an animal testing alternative. The chick embryonic heart develops through a process similar to that in mice, rats, and humans and has a similar atrioventricular system. ${ }^{17)}$ Accordingly, the cardiotoxicity induced by amitriptyline could be clearly analyzed in chick embryos, as in mammals, especially the decrease in heart rate accompanied by prolongation of the QTc interval, as was observed in humans. The clinical use of amitriptyline also results in cardiotoxicity. ${ }^{5,6,18,19)}$ This indicates that developing chick embryos are appropriate as an alternative experimental animal to replace the traditional mammals. Although the exact mechanism of amitriptyline cardiotoxicity remains to be clarified, the drug appears to induce it in chick embryos.

It is known that amitriptyline exhibits circadian stage-dependent toxicity in humans. ${ }^{20)}$ We previously used chick embryos as an experimental model to evaluate the chronopharmacology and chronopharmacokinetics of drugs. ${ }^{21-23)}$ In the present study, the light schedule modified the toxicity of amitriptyline in chick embryos. The light schedule appears to be an important factor in clarifying the circadian rhythm of drug actions. In mice housed under standardized light-dark conditions, there was a highly significant circadian rhythm in mortality depending on the time of drug administration, although the circadian rhythm of the toxicity disappeared in 
mice housed under constant dark conditions. ${ }^{24)}$

One might wonder whether different light/dark conditions might affect the development of incubated embryos. In the untreated state, the heart rate of the chick embryos did not differ significantly under light and dark conditions, and body weights increased with the day of incubation. These findings indicate that the manipulation of the light schedule markedly influences the toxicity of amitriptyline in chick embryos. In the chicken, the pineal body contains an endogenous oscillator and a photoreceptor for circadian rhythmicity. ${ }^{25)}$ Although the exact mechanism underlying the influence of the light schedule on the toxicity of amitriptyline remains to be clarified, dark condition appear to enhance the toxicity of this drug in chick embryos.

In conclusion, our ECG recording system for chick embryos may be useful for investigating the toxicity of tricyclic antidepressants. In addition, the manupilation of the light schedule and the timing of drug actions in relation to these lighting conditions may help us to design rational chronopharmacology for some drugs, including tricyclic antidepressants, under certain experimental situations.

Acknowledgments The authors express their gratitude to Ms. Takako Suzuki and Ms. Yayoi Mihara for their assistance in this study.

This study was supported in part by the Japanese Society of Alternatives to Animal Experiments (JSAAE).

\section{REFERENCES}

1) Yoshiyama Y., Kobayashi T., Hodo S., Ogawa N., Bergeron M., Labrecque G., Beauchamp D., Nakano S., J. Infect. Chemother., 2, 106-109 (1996).

2) Yoshiyama Y., Nakano S., Ogawa N., J. Clin. Pharmacol., 29, $1048-$ 1052 (1989).

3) Yoshiyama Y., Grenier L., Gourde P., Simard M., Lin L., Morin N.,
Bergeron M., Labrecque G., Beauchamp D., Antimicrob. Agents Chemother, 40, 802-806 (1996).

4) Yoshiyama Y., Nishikawa S., Sugiyama T., Kobayashi T., Shimada H., Tomohaga F., Ohdo S., Ogawa N., Nakano S., Antimicrob. Agents Chemother, 37, 2042-2043 (1993).

5) Robinson D. S., Barker E., JAMA, 236, 2089-2090 (1976).

6) Biggs J. T., Spiker D. G., Petit J. M., JAMA, 238, 135-138 (1977).

7) Tazawa H., Yamaguchi S., Yamada M., Doi K., Comp. Biochem. Physiol., 101A, 103-108 (1992).

8) Rajala G. M., Kuhlmann R. S., Kolesari G. L., Teratology, 30, 385392 (1984).

9) Paff G. H., Boucek R. J., Anat. Rec., 182, 169-173 (1975).

10) Yoshiyama Y., Sugiyama T., Kanke M., Biol. Pharm. Bull., 26, 893895 (2003).

11) Yoshiyama Y., Sugiyama T., Kanke M., AATEX, 9, 40-44 (2003).

12) Miyazaki H., Sugiyama T., Saito K., Kubota N., Yoshiyama Y., Shimada H., In Vitro Toxicol., 7, 243-246 (1994).

13) Saito K., Sugiyama T., Shimada H., Eur. J. Pharmacol., 183, 22362237 (1990).

14) Sugiyama T., Miyazaki H., Saito K., Shimada H., Miyamoto K., Toxicol. Appl. Pharmacol., 138, 262-267 (1996).

15) Product Information: Elavil ${ }^{\circledR}$, amitriptyline. Merck \& Co., Inc., West Point, PA, (PI revised 12/1999) reviewed 1/2003 (2003).

16) Bigger J. T., Giardino C. G., Perel J. M., N. Engl. J. Med., 296, $206-$ 208 (1977).

17) Bulter H., Juurlink B. H. J., "An Atlas for Staging Mammalian and Chick Embryos," CRC Press, Boca Raton, 1987, pp. 19-138.

18) Tobis J., Das B. N., JAMA, 234, 1474-1476 (1976).

19) Wright S. P., Clin. Pediatr., 15, 1123-1128 (1976).

20) Nakano S., Hollister L. E., Clin. Pharmacol. Ther, 33, 453-459 (1983).

21) Yoshiyama Y., Sugiyama T., Kanke M., Tsuchimoto K., Biol. Pharm. Bull., 24, 429-431 (2001).

22) Yoshiyama Y., Sugiyama T., Miyazaki H., Shimada H., Ohdo S., AATEX, 4, 55-61 (1997).

23) Yoshiyama Y., Sugiyama T., Kubota N., Miyazaki H., Saito K., Tomonaga F., Shimada H., Ohdo S., Biol. Pharm. Bull., 18, 776-778 (1995).

24) Nakano S., Song J., Ogawa N., Ann. Rev. Chronopharmacol., 7, 277280 (1990).

25) Deguchi T., Nature (London), 282, 94—98 (1979). 